\title{
Particle swarm optimization based optimal bidding strategy in an open electricity market
}

\author{
J. Vijaya Kumar ${ }^{1 *}$, D.M. Vinod Kumar ${ }^{2}$ \\ ${ }^{1 * 2}$ Department of Electrical Engineering, National Institute of Technology Warangal, INDIA \\ "Corresponding Author: e-mail: jvkeee@gmail.com, Tel: +91-9290852689, Fax: +91-870-2459547
}

\begin{abstract}
In an electricity market generating companies and large consumers need suitable bidding models to maximize their profits. Therefore, each supplier and large consumer will bid strategically for choosing the bidding coefficients to counter the competitors bidding strategy. In this paper, bidding strategy problem modeled as an optimization problem and solved using Particle Swarm Optimization (PSO). PSO shares many similarities with evolutionary computation techniques such as Genetic Algorithm (GA). The system is initialized with a population of random solutions and searches for optima by updating generations. However, unlike GA, PSO has no evolution operators such as crossover and mutation. In PSO, the potential solutions, called particles, fly through the problem space by following the current optimum particles. A numerical example with six suppliers and two large consumers is used to illustrate the essential features of the proposed method and the results are compared with the Genetic Algorithm (GA) approach. Test results indicate that the proposed algorithm outperforms the Genetic Algorithm approach with respect to total profit and convergence time.
\end{abstract}

Keywords: Electricity Market, Market Clearing Price (MCP), Optimal bidding strategy, Particle Swarm Optimization (PSO).

DOI: http://dx.doi.org/10.4314/ijest.v3i6.23

\section{Introduction}

Restructuring of the power industry mainly aims at abolishing the monopoly in the generation and trading sectors, thereby, introducing competition at various levels wherever it is possible. But the sudden changes in the electricity markets have a variety of new issues such as oligopolistic nature of the market, supplier's strategic bidding, market power misuse, price-demand elasticity and so on. Theoretically, in a perfectly competitive market, supplier should bid at their marginal production cost to maximize payoff. However, practically the electricity markets are oligopolistic nature, and power suppliers may seek to increase their profit by bidding a price higher than marginal production cost. Knowing their own costs, technical constraints and their expectation of rival and market behavior, suppliers face the problem of constructing the best optimal bid. This is known as a strategic bidding problem.

A comprehensive review of optimal bidding strategies in Electricity Market (EM) has been published (David et al, 2000). Strategic bidding problem (David et al, 2000) is solved using Dynamic Programming (DP) based approach. A Lagrangian relaxation-based approach for strategic bidding in England-Wales pool type electricity market has been adopted (Gross et al, 1996). The same approach for daily bidding and self-scheduling decision in New England market has been suggested (Zhang et al, 1999). A considerable amount of work has also been reported on the game theory applications in the competitive electricity market. (Ebrahim et al, 2010) developed Nash equilibrium based bidding strategy in a competitive electricity market. In noncooperative game theory approach (Ferrero et al, 1997, Torre et al, 2004), strategic bidding problem was solved using Nash equilibrium. (Jainhui et al, 2011) used evolutionary game approach to analyzing bidding strategies by considering elastic demand. Genetic Algorithm (GA) has been proposed (David et al, 2001) to develop an overall bidding strategy using two different bidding schemes for a day-ahead market. The same methodology has been extended for spinning reserve market coordinated with energy market (David et al, 2002). (Azadeh et al, 2011) formed optimal bidding problem for a day-ahead market as a multi objective problem and solved using GA. (Ugedo et al, 2006) have proposed a stochastic-optimization approach for submitting the block bids 
in sequential energy and ancillary services markets, and uncertainty in demand and rival's bidding behaviour is estimated by stochastic residual demand curves based on decision trees.

A stochastic programming model has been used to construct linear bid curves in the Nord-pool market for price-taking retailer whose customers' load is price flexible (Fleten et al, 2005). Opponents' bidding behaviours are represented as a discrete probability distribution function (Song et al, 1999) and as a continuous probability distribution function (David et al, 2001) for a supplier's bid decision-making problem. Strategic bidding problem has been formulated as a two level optimization problem in which producers try to maximize their profit based on the market clearing price (or bid price), and dispatch quantity is obtained from an optimal power flow model. Using deterministic approach, it is difficult to obtain the global solution of such bi-level optimization problem because of non-convex objective functions and non-linear complementarity conditions (Hobbs et al, 2004), to represent market clearing. These difficulties are avoided by representing the residual demand function by Mixed Integer Linear Programming (MILP) model (Torre et al, 2002), in which unit commitment and uncertainties are also taken into account. (Pereira et al, 2005), the generators associated to the competitors' firms have been explicitly modelled as an alternative MILP formulation based on a binary expansion of the decision variables (price and quantity bids). Risk constrained bidding strategy for single sided and double sided bidding is solved using GA (Jain et al, 2009]. PSO is used to determine bid prices and quantities under the rules of a competitive power market (Ahmet et al, 2009). Strategic bidding for pumped-storage hydroelectric plant using evolutionary tristate PSO is developed by (Kanakasabhapathy et al, 2010).

In general, strategic bidding is an optimization problem that can be solved by various conventional and nonconventional (heuristic) methods. Depending on the bidding models, objective function and constraints may not be differentiable and then conventional methods cannot be applied. Heuristic methods such as GA, Simulated Annealing (SA) and Evolutionary Programming (EP) etc, have main limitations of their sensitivity to the choice of parameters, such as crossover and mutation probabilities in GA, temperature in SA, scaling factor in EP. PSO is a modern stochastic search algorithm and a kind of evolutionary computation technique (Kennedy et al, 1995). The major difference between PSO and other evolutionary algorithms is that, in PSO, the particles remain the same, but their characteristics (e.g. position and velocity) change, where as in EP, the individuals in population change, with new individuals being 'generated' in each iteration. The PSO technique can generate better quality solution within shorter calculation time and stable convergence characteristic than other stochastic methods such as GA, EP etc.

The main contribution of this paper is a new optimization paradigm based on Particle Swarm Optimization (PSO) is applied to solve optimal bidding strategy problem. The result shows that the proposed algorithm can generate better quality solution within shorter computation time and stable convergence characteristics compared to GA. The paper is organized as follows. Section 2 presents the mathematical formulation of optimal bidding problem. Section 3 contains a brief over view of the proposed PSO method and application of PSO for solving the optimal bidding problem. Section 4 reports the case studies solving optimal bidding problem for the given data and Section 5 summed up the final outcome of the paper as Conclusion.

\section{Problem Formulation}

Consider a system consist of ' $m$ ' Generators an inter-connected network controlled by an ISO, a Power Exchange (PX), an aggregated consumer (load) which does not participate in demand-side bidding but is elastic to the price of electricity, and ' $n$ ' large consumers who participate in demand-side bidding. The supplier and large consumer is required to bid a linear nondecreasing supply and non-increasing demand function to PX, bid linear supply curve denoted by $G_{i}\left(P_{i}\right)=a_{i}+b_{i} P_{i}$ when $i=$ $1,2, \ldots . . m$ and for large consumers bid linear demand curve denoted by $W_{j}\left(L_{j}\right)=c_{j}-d_{j} L_{j}$ when $j=1,2, \ldots . n$. Here $P_{i}$ is the active power output, $a_{i}$ and $b_{i}$ the bidding coefficients of the $i^{t h}$ supplier. $L_{j}$ is the active power load, $c_{j}$ and $d_{j}$ the bidding coefficients of the $j^{\text {th }}$ large consumer; $a_{i}, b_{i}, c_{j}$ and $d_{j}$ are non-negative.

The main function of PX is to determine a generation/demand dispatch/schedule that meets security and reliability constraints using transparent dispatch procedures, with the objective of maximizing payoff. Moreover, when the suppliers and large consumers bid linear supply and demand functions and the network constraints are ignored, maximizing payoff leads to a uniform market clearing price for all suppliers and consumers. Thus, when only the load flow constraints and generation output limit and consumer demand limit constraints are considered, $\mathrm{PX}$ determines a set of generation outputs $\mathrm{P}=\left(\mathrm{P}_{1}, \mathrm{P}_{2} \ldots \mathrm{P}_{\mathrm{m}}\right)^{\mathrm{T}}$ and a set of large consumers' demands $\mathrm{L}=\left(\mathrm{L}_{1}, \mathrm{~L}_{2}, \ldots . \mathrm{L}_{n}\right)^{\mathrm{T}}$ by solving equations (1) to (5).

$$
\begin{aligned}
& a_{i}+b_{i} P_{i}=R \quad i=1,2 \ldots m \\
& c_{j}-d_{j} L_{j}=R \quad j=1,2 \ldots \ldots n \\
& \sum_{i=1}^{m} P_{i}=Q(R)+\sum_{j=1}^{n} L_{j}
\end{aligned}
$$




$$
\begin{gathered}
P_{\min , i} \leq P_{i} \leq P_{\max , i} \quad i=1,2 \ldots \ldots m \\
L_{M I N, J} \leq L_{j} \leq L_{\max , j} \quad j=1,2 \ldots \ldots . n
\end{gathered}
$$

$R$ is the uniform market clearing price of electricity to be determined. $Q(R)$ is the aggregate pool load forecast by PX and made known to all participants and is assumed to be dependent on the price of electricity. $P_{\min , i}$ and $P_{\max , i}$ are the generation output limits of the $i^{\text {th }}$ supplier, and $L_{\min , j}$ and $L_{\max , j}$ are the demand limits of the $j^{\text {th }}$ large consumer. The expression for $Q(R)$ is available; equations (1) to (3) can be solved directly. The procedure is basically the same as that for the classical economic dispatch problem (Wood et al, 1995). Suppose the aggregate pool load $Q(R)$ takes the following linear form:

$$
Q(R))=Q_{o}-K R
$$

where $Q_{o}$ is a constant number and $K$ is a coefficient denoting the price elasticity of the aggregate demand. If pool demand is largely inelastic, then $K=0$. The inequality constraints (4) and (5) are ignored, the solutions to equations (1) to (3) are

$$
\begin{gathered}
R=\frac{Q_{o}+\sum_{i=1}^{m} \frac{a_{i}}{b_{i}}+\sum_{j=1}^{n} \frac{c_{j}}{d_{j}}}{K+\sum_{i=1}^{m} \frac{1}{b_{i}}+\sum_{j=1}^{n} \frac{1}{d_{j}}} \\
P_{i}=\frac{R-a_{i}}{b_{i}} \quad i=1,2 \ldots \ldots \ldots \ldots m \\
L_{j}=\frac{c_{j}-R}{b_{j}} \quad j=1,2 \ldots \ldots \ldots \ldots n
\end{gathered}
$$

When the solution set (8) and (9) violates generation output/consumer demand limits (4) and (5), it must be modified to accommodate these limits. For the $i^{\text {th }}$ supplier has the cost function denoted by $C_{i}\left(P_{i}\right)=e_{i} P_{i}+f_{i} P_{i}^{2}$, here $e_{i}$ and $f_{i}$ are cost function coefficients. The benefit maximization objective for building a bidding strategy can be described as:

$$
\begin{aligned}
& \text { Maximize: } F\left(a_{i}, b_{i}\right)=R P_{i}-C_{i}\left(P_{i}\right) \\
& \text { Subject to: (1) to (5) }
\end{aligned}
$$

The objective is to determine $a_{i}$ and $b_{i}$ so as to maximize $F\left(a_{i}, b_{i}\right)$ subject to the constraints (1) - (5). $C_{i}\left(P_{i}\right)$ is the production cost function of the $i^{\text {th }}$ supplier. Similarly, for the $j^{\text {th }}$ large consumer has revenue function $B_{j}\left(L_{j}\right)=g_{j} L_{j}-h_{j} L_{j}^{2}$, here $g_{j}$ and $h_{j}$ are the demand function coefficients. The benefit maximization objective for building a bidding strategy can be described as:

$$
\begin{aligned}
& \text { Maximize: } B\left(c_{j}, d_{j}\right)=B_{j}\left(L_{j}\right)-R L_{j} \\
& \text { Subject to: (1) to (5) }
\end{aligned}
$$

The objective is to determine $c_{j}$ and $d_{j}$ so as to maximize $B\left(c_{j}, d_{j}\right)$ subject to the constraints (1) to (5). $B_{j}\left(L_{j}\right)$ is the demand (benefit) function of the $j^{\text {th }}$ large consumer. In the sealed bid auction based electricity market, data for the next bidding period is confidential, and hence suppliers/large consumers do not have the information needed to solve the optimization problem (10) and (11). However, the past bidding histories are available, and estimation of the bidding coefficients of rivals is possible. An immediate problem for each participant is how to estimate the bidding coefficients of rivals.

Let from the $i^{\text {th }}$ supplier's point of view, rival's $j^{\text {th }}(j \neq i)$ bidding coefficients obey a joint normal distribution with the following probability density function $(p d f)$ : 


$$
\begin{aligned}
& p d f_{i}\left(a_{j}, b_{j}\right)=\frac{1}{2 \Pi \sigma_{j}^{(a)} \sigma_{j}^{(b)} \sqrt{1-\rho_{j}^{2}}} \\
& \quad \times \exp \left\{-\frac{1}{2\left(1-\rho_{j}^{2}\right)}\left[\left(\frac{a_{j}-\mu_{j}^{(a)}}{\sigma_{j}^{(a)}}\right)^{2}-\frac{2 \rho_{j}\left(a_{j}-\mu_{j}^{(a)}\right)\left(b_{j}-\mu_{j}^{(b)}\right)}{\sigma_{j}^{(a)} \sigma_{j}^{(b)}}+\left(\frac{b_{j}-\mu_{j}^{(b)}}{\sigma_{j}^{(b)}}\right)^{2}\right]\right\}
\end{aligned}
$$

where ' $\rho_{j}$ ' is the correlation coefficient between $a_{j}$ and $b_{j}$, and $\mu_{j}^{(a)}, \mu_{j}^{(b)} \sigma_{j}^{(a)}$ and $\sigma_{j}^{(b)}$ are the parameter of the joint distribution. The marginal distributions of $a_{j}$ and $b_{j}$ are both normal with mean values $\mu_{j}^{(a)}$ and $\mu_{j}^{(b)}$, and standard deviations $\sigma_{j}^{(a)}$ and $\sigma_{j}^{(b)}$ respectively. Similarly, the above probability density function ( $p d f$ ) is also used for finding bidding coefficients of the large consumers. Based on historical bidding data these distributions can be determined (David et al, 2001). Using probability density function (12) for supplier as well as large consumers the joint distribution between $a_{j}$ and $b_{j}$, and between $c_{j}$ and $d_{j}$, the optimal bidding problem with objective functions given in equation (10) and (11) and constraints (1) to (5) becomes a stochastic optimization problem. In this paper, PSO is used, which is very efficient to solve the above stochastic optimization problem, presented in the following section.

\section{Particle Swarm Optimization (PSO)}

The PSO method is a self-educating optimization algorithm that can be applied to any nonlinear optimization problem (Kennedy et al, 1995). In PSO, the potential solutions, called particles, fly through the problem space by following the best fitness of the particles. It exhibits some evolutionary computation attributes such as initialization with a population of random solutions and search for optima by updating generations. The updates of particles are accomplished according to the following equations. Equation (13) calculates a new velocity for each particle $r$, based on its previous velocity $\left(V_{r} k\right)$, the particle's location at which the best fitness has been achieved $\left(P_{\text {best }} r\right)$ so far, and the best particle among the neighbors $\left(G_{\text {best }}\right)$ at which the best fitness has been achieved so far. The learning factors $a_{1}$ and $a_{2}$ are the acceleration constants that change the velocity of a particle towards $P_{b e s t} r$ and $G_{\text {best }}$, and rand $_{1}$, rand $_{2}$ are uniformly distributed random numbers in [0,1]. Each particle's position is updated using (14) in the solution hyperspace. It is concluded (Singh $e t a l$, 2008) that the PSO with a Linearly Decreasing (LD) inertia weight $w^{k}$ in each iteration $k$, from maximum value $w_{\max }$ to minimum value $w_{\min }$, as reflected in (15) can make a significant improvement on convergence to the global optimum within a reasonable number of iterations.

$$
\begin{aligned}
& V_{r}^{k+1}=w^{k} V_{r}^{k}+a_{1} \text { rand }_{1} *\left(P_{\text {best }}^{k}-X_{r}^{k}\right)+a_{2} \text { rand }_{2} *\left(G_{\text {best }}^{k}-X_{r}^{k}\right) \\
& X_{r}^{k+1}=X_{r}^{k}+V_{r}^{k+1} \\
& w^{k}=w_{\max }-\frac{w_{\max }-w_{\min }}{k_{\max }} * k
\end{aligned}
$$

where $k$ is the iteration counter and $k_{\max }$ is the maximum iteration number.

The velocity update expression (13) can be explained as follows (Yoshida et al, 2000). Without the second and third terms, the first term (representing inertia) will keep a particle flying in the same direction until it hits the boundary. Therefore the first term tries to explore new areas and corresponds to the diversification in the search procedure. In contrast, without the first term, the velocity of the flying particle is only determined by using its current position and its best positions in history. Therefore the second (representing memory) and third term (representing cooperation) try to converge the particles to their $P_{\text {best } r}$ and/or $G_{\text {best }}$ and correspond to the intensification in the search procedure. Namely, the PSO has a well-balanced mechanism to utilise the diversification and the intensification in the search procedure efficiently.

3.1 PSO Algorithm for Bidding Problem: It is obvious that for maximizing the benefits of a supplier or large consumer, both members of the pair coefficients $\left(a_{i}, b_{i}\right)$ and $\left(c_{j}, d_{j}\right)$ cannot be selected independently in other words, a supplier or large consumer can fix one of these two coefficients and then determine the other by using an optimization procedure. In this regard, PSO is applied to find the optimal bidding coefficients and profit of each participant. The algorithms are as follows. 
(a) PSO for obtaining optimal bidding coefficients $\left(b_{i} / d_{j}\right)$

Step1. Initialization of the particles

(a). Generate random population of $b_{i}$ solutions //where $b_{i}$ is the bidding parameter of the $i^{\text {th }}$ supplier to be optimized//.

(b). Read input data $\mu, \sigma, \rho, a$ and maximum iterations. // where $\mu=$ mean, $\sigma=$ standard deviation, $\rho=$ correlation coefficient of (12), $a=$ cost coefficient //

Step2. The evaluation function of each individual $b_{i}$ is calculated in the population using (12). Here $p d f$ is Fitness evaluation.

Step3. Each $P_{b e s t}$ values are compared with the other $P_{\text {best }}$ values in the population. The best evaluation value among the $P$-bests is denoted as $G_{\text {best }}$.

Step4. The member velocity $V$ of each individual $b_{i}$ is modified according to the velocity update (13)

Step5. The position of each individual $b_{i}$ is modified according to the position update (14)

Step6. Repeat from steps 2-5 until iteration reaches their maximum limit. Return the best fitness (optimal bid value $b_{i}$ ) computed at final iteration as a global fitness. Using $b_{i}$ values, calculate MCP from (7).

A similar procedure is applied to find the optimal values of $d_{j}$.

(b) Maximization of profit for supply-side biding using PSO

Step1. Initialization of the particles

(a). Generate random population of profit $F_{j}\left(a_{i}, b_{i}\right)$ solutions //where $F_{j}$ is the profit of the $j^{\text {th }}$ supplier//.

(b). Read input data of Generators (i.e. cost coefficients, $\left.P_{\min }, P_{\max }\right)$, demand $\left(Q_{o}\right)$ and maximum number of iterations.

Step2. Calculate generator output each supplier using (8)

(a) If generation violates lower limit set as a lower limit If generation violates upper limit set as an upper limit

(b) Add all generations

(c) Error = total system generation - total system demand

Step3. Fitness evaluation using (10) and (11)

Step4. Each $P_{\text {best }}$ values are compared with the other $P_{\text {best }}$ values in the population. The best evaluation value among the $P$-bests is denoted as Gbest.

Step5. The member velocity $V$ of each individual $b_{i}$ is modified according to the velocity update (13)

Step6. The position of each individual $b_{i}$ is modified according to the position update (14)

Step7. Repeat from steps 3- 6 until iteration reaches their maximum limit. Return the best fitness (maximum profit) computed at final iteration as a global fitness.

Step9. Print c.p.u time and plot number of iterations versus \% Error.

$$
\% \text { Error }=\frac{\text { Generation }- \text { Demand }}{\text { Generation }} \times 100
$$

PSO uses random initialisation, but it gives almost the same optimal solution in a set of simulations within a given case. It shows its immunity to the start point. Updating inertia weight according to (15) is known as Inertia Weighted Approach (IWA). By Linearly Decreasing (LD), the inertia weight from a relatively large value to a small value through the course of PSO run, the PSO tends to have more global search ability at the beginning of the run while having more local search ability near the end of the run. Hence, IWA provides a balance between global and local explorations. The number of maximum iterations required to obtain the global solution is dependent on the nature and the size of the problem. In general, the maximum iteration depends upon the evolving process, which provides dynamic information of convergence. Figure 1 illustrates the flowchart of the PSO to solve the optimal bidding problem. 


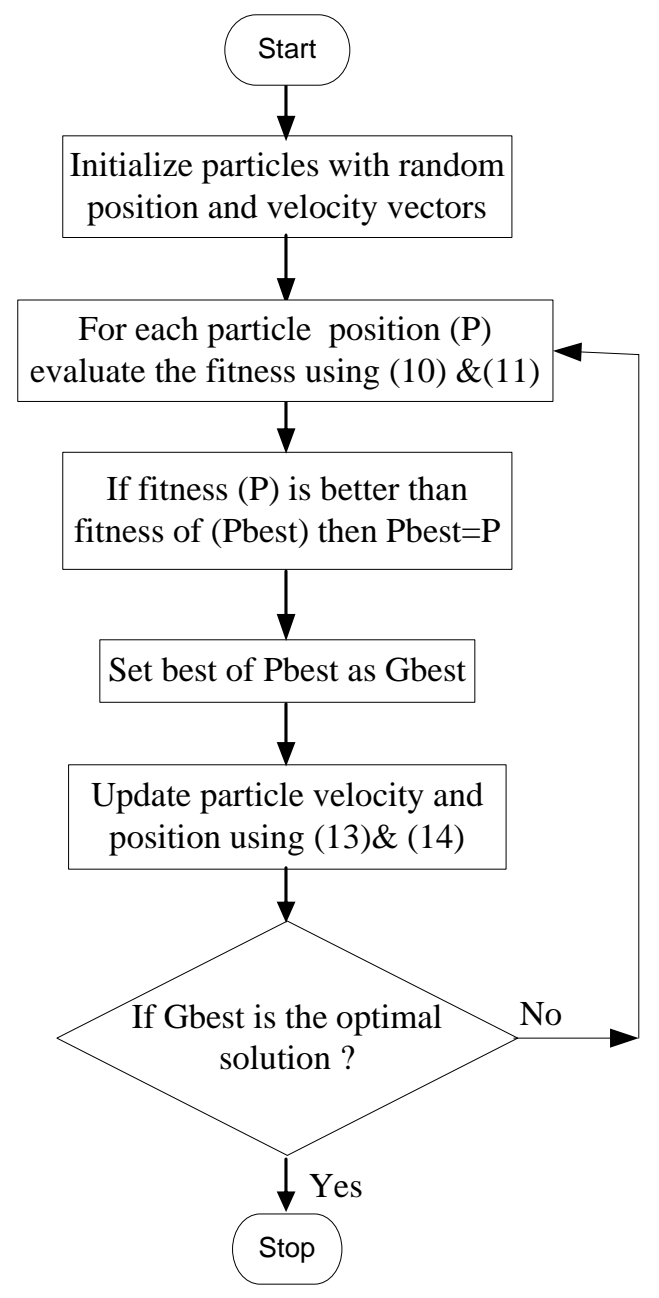

Figure 1 Flowchart of the proposed PSO algorithm.

\section{Results and Discussions}

Consider a system consists of six suppliers, who supply electricity aggregate load and two large consumers. The Generator and large consumer data are shown in Table $1 . Q_{o}$ is 300 and $K$ is 5 for aggregated load. In this work, the parameters used after fine tuning for PSO and GA are, Population size: 50, accelerating factors $a l=a 2: 2.0$, inertia weight $w: 1.0$ to 0.5 , Maximum number of iterations: 150 for PSO; Population size: 50, Maximum number of iterations: 150, Elitism probability $\left(P_{e}\right)$ : 0.15 , Crossover Probability $\left(P_{c}\right): 0.85$, Mutation probability $\left(P_{m}\right): 0.005$ for GA; Simulations are carried on 2.66GHz, PIV Processor, 3GB RAM and MATLAB 7.8 version is used.

Table1. Generator and Large Consumer Data

\begin{tabular}{|c|c|c|c|c|}
\hline Generator & $e$ & $f$ & $P_{\min }(M W)$ & $P_{\max }(M W)$ \\
\hline 1 & 6.0 & 0.01125 & 40 & 160 \\
\hline 2 & 5.25 & 0.0525 & 30 & 130 \\
\hline 3 & 3.0 & 0.1375 & 20 & 90 \\
\hline 4 & 9.75 & 0.02532 & 20 & 120 \\
\hline 5 & 9.0 & 0.075 & 20 & 100 \\
\hline 6 & 9.0 & 0.075 & 20 & 100 \\
\hline Large Consumer & $g$ & $h$ & $L_{\min }(M W)$ & $L_{\max }(M W)$ \\
\hline 1 & 30 & 0.04 & 0 & 200 \\
\hline 2 & 25 & 0.03 & 0 & 150 \\
\hline
\end{tabular}


4.1 Case A: With symmetrical information: Each rival participant is assumed to have an estimated joint normal distribution for the two bidding coefficients. For the $p^{\text {th }}$ participant $(p=1,2, \ldots, 8)$, the estimated parameters in the joint normal distributions for the $i^{\text {th }}$ supplier $(i=1,2, \ldots, 6$ and $i \neq p)$ and for the $j^{\text {th }}$ large consumer $(j=1,2$ and $j+6 \neq p)$, as described in (12) are specified in PSO as (16) and (17):

$$
\begin{gathered}
\mu_{i}^{(a)}=1.2 e_{i} \quad \mu_{i}^{(b)}=1.2 \times 2 f_{i} \\
4 \sigma_{i}^{(a)}=0.15 e_{i} \quad 4 \sigma_{i}^{(b)}=0.15 f_{i} \\
\rho_{i}=-0.1 \\
\mu_{j}^{(c)}=1.2 g_{j} \quad \mu_{j}^{(d)}=1.2 \times 2 h_{j} \\
4 \sigma_{j}^{(c)}=0.15 e_{j} \quad 4 \sigma_{j}^{(b)}=0.15 f_{j} \\
\gamma_{j}=0.1
\end{gathered}
$$

It should be mentioned that the parameters in (16) and (17) should be estimated using available mathematical methods such as the one in based on sufficient historical bidding data. Since we do not have such data, we are unable to determine these parameters mathematically, and instead, these parameters are specified here to illustrate the basic feature of the method and these specifications may not well reflect practical situations. Some explanation about the specifications of parameters in (16) and (17) is necessary. It is a reasonable assumption that a supplier who is aware of market power in the reformed electricity market is likely to bid above the production cost. Hence, the expected values of $a_{i}$ and $b_{i}$, i.e. $\mu_{i}^{(a)}$ and $\mu_{i}^{(b)}$ are specified 20\% above $e_{i}$ and $2 f_{i}$, respectively. The standard deviations of $a_{i}$ and $b_{j}$, i.e. $\sigma_{i}^{(a)}, \sigma_{i}^{(b)}$ are specified to make $a_{i}$ and $b_{i}$ fall in the domains [1.05e $e_{i}, 1.35 e_{i}$ ] $=\left[\mu_{i}^{(a)} 4 \sigma_{i}^{(a)} \mu_{i}^{(a)} 4 \sigma_{i}^{(a)}\right]$ and [1.05×2f, $\left.1.35 \times 2 f_{i}\right]=\left[\mu_{i}^{(b)} 4 \sigma_{i}^{(b)} \mu_{i}^{(b)} 4 \sigma_{i}^{(b)}\right.$ ] respectively, with probability 0.9999. $\rho_{i}$ is specified to be negative because when a supplier decides to increase one of his or her bidding coefficients, it is more likely that, in a mature market, it will decrease rather than increase the other coefficient ( David et al, 2001).

A similar explanation is applicable to the specifications of parameters in (17), by using PSO, bidding coefficients, generation outputs; market clearing price and profit of six suppliers and two large consumers are calculated. The same problem is also solved by using GA and compared with Monte Carlo method (David et al, 2001) shown in Table 2 and Table 3. Table 2 shows the optimal bidding parameters and Table 3 shows the MCP and profit of each supplier. From the Table 3, it is observed that the profits obtained by each supplier is more, when compared with GA and Monte Carlo method, therefore the bidding strategies obtained by PSO are optimum compared to GA and Monte Carlo method. Convergence characteristics are shown in Figure 2 for the proposed PSO and GA method. From the Figure 2 it is observed that PSO converges much faster compared to GA because, the main limitations of GA are selection of crossover and mutation probabilities. Where as in the case of PSO there is no selection parameter. The major difference between PSO and GA is that, in PSO, the particles remain the same, but their characteristics (e.g. position and velocity) change, where as in GA, the individuals in population change, with new individuals being 'generated' in each iteration. Therefore PSO technique can generate better quality solution within shorter calculation time and stable convergence characteristic than GA. Figure 3 gives the variation of profit of all participants (i.e. suppliers as well as consumers) and Figure 4 gives the expected dispatch powers of suppliers for the proposed method and GA. The result shows that the proposed method is more efficient and superior than GA. 
Table 2. Bidding Strategies of Generators and Consumers

\begin{tabular}{|c|c|c|c|}
\hline & PSO & GA & $\begin{array}{c}\text { Monte Carlo (David } \\
\text { et al, 2001) }\end{array}$ \\
\hline Generator & $b_{i}$ & $b_{i}$ & $b_{i}$ \\
\hline 1 & 0.062 & 0.058 & 0.0297 \\
\hline 2 & 0.079 & 0.101 & 0.124 \\
\hline 3 & 0.243 & 0.221 & 0.292 \\
\hline 4 & 0.046 & 0.035 & 0.074 \\
\hline 5 & 0.124 & 0.116 & 0.170 \\
\hline 6 & 0.124 & 0.116 & 0.170 \\
\hline Large Consumer & $d_{j}$ & $d_{j}$ & $d_{j}$ \\
\hline 7 & 0.072 & 0.064 & 0.097 \\
\hline 8 & 0.051 & 0.049 & 0.077 \\
\hline
\end{tabular}

Table 3. Bid Price (\$/MWh) and Profit (\$) of Generators and consumers

\begin{tabular}{|c|c|c|c|c|c|c|}
\hline & \multicolumn{2}{|c|}{ PSO } & \multicolumn{2}{c|}{ GA } & \multicolumn{2}{c|}{$\begin{array}{c}\text { Monte Carlo } \\
\text { (David et al, 2001) }\end{array}$} \\
\hline Generator & P (MW) & Profit & P (MW) & Profit & P (MW) & Profit \\
\hline 1 & 156.00 & 1320.3 & 152.00 & 1310.1 & 160.00 & 1368.0 \\
\hline 2 & 104.38 & 574.1 & 102.83 & 504 & 89.4 & 572.7 \\
\hline 3 & 47.271 & 316.2 & 41.921 & 291.8 & 45.7 & 322.9 \\
\hline 4 & 119.380 & 416.1 & 116.28 & 384.7 & 88.8 & 386.4 \\
\hline 5 & 48.762 & 178.4 & 46.025 & 165.8 & 43.1 & 177.5 \\
\hline 6 & 48.762 & 178.4 & 46.025 & 165.8 & 43.1 & 177.5 \\
\hline Large consumer & L (MW) & Profit & L(MW) & Profit & L(MW) & Profit \\
\hline 7 & 168.97 & 1146 & 162.61 & 1135.6 & 139.7 & 1126.3 \\
\hline 8 & 140.92 & 611.8 & 139.95 & 596.4 & 112.1 & 592.6 \\
\hline MCP & 16.47 & 15.81 & \multicolumn{2}{c|}{16.35} \\
\hline Total Profit & 4741.3 & 4554.2 & 4723.9 \\
\hline
\end{tabular}

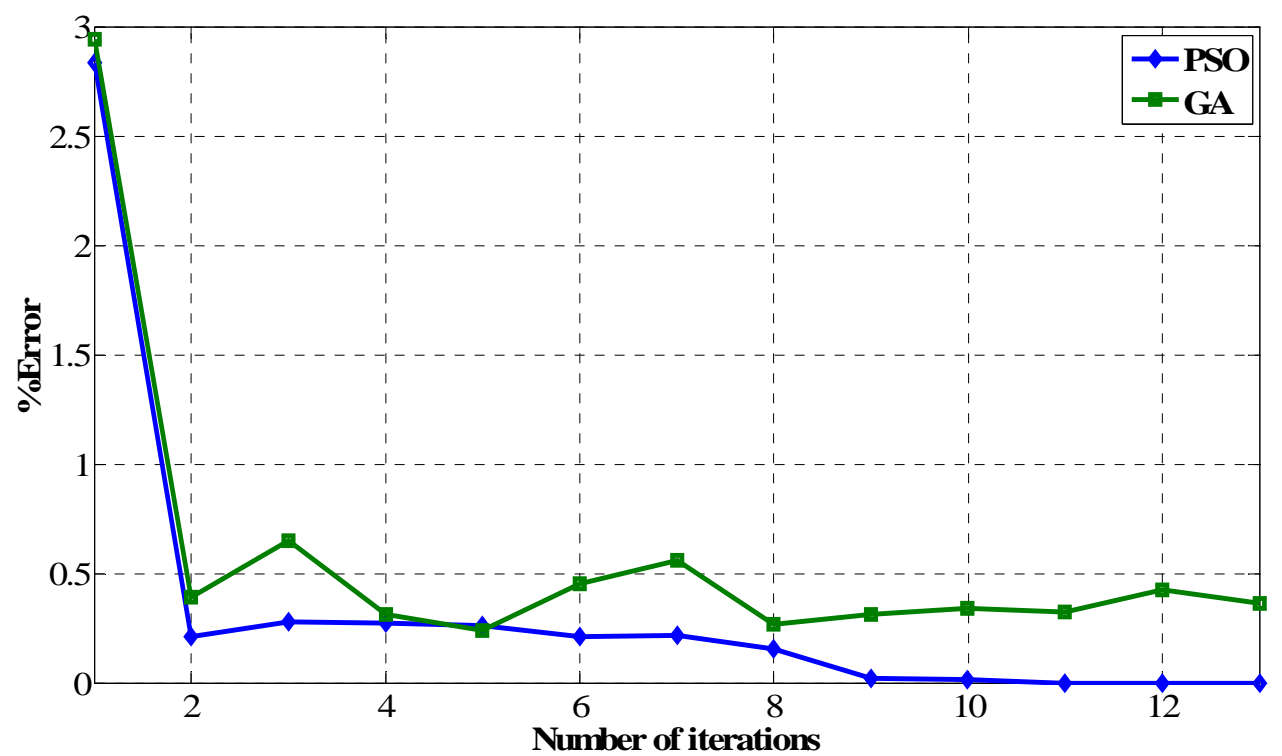

Figure 2 Convergence characteristics of PSO and GA 


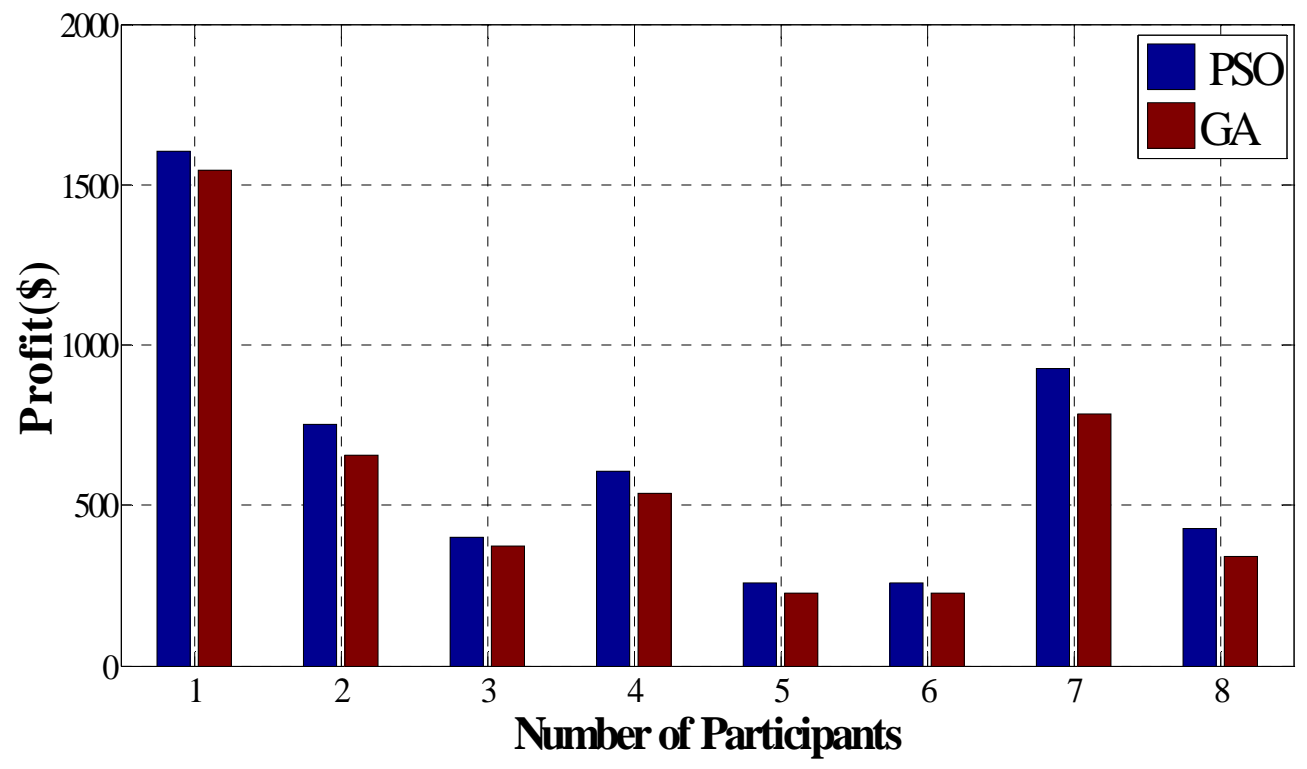

Figure 3 Expected profits of suppliers and consumers

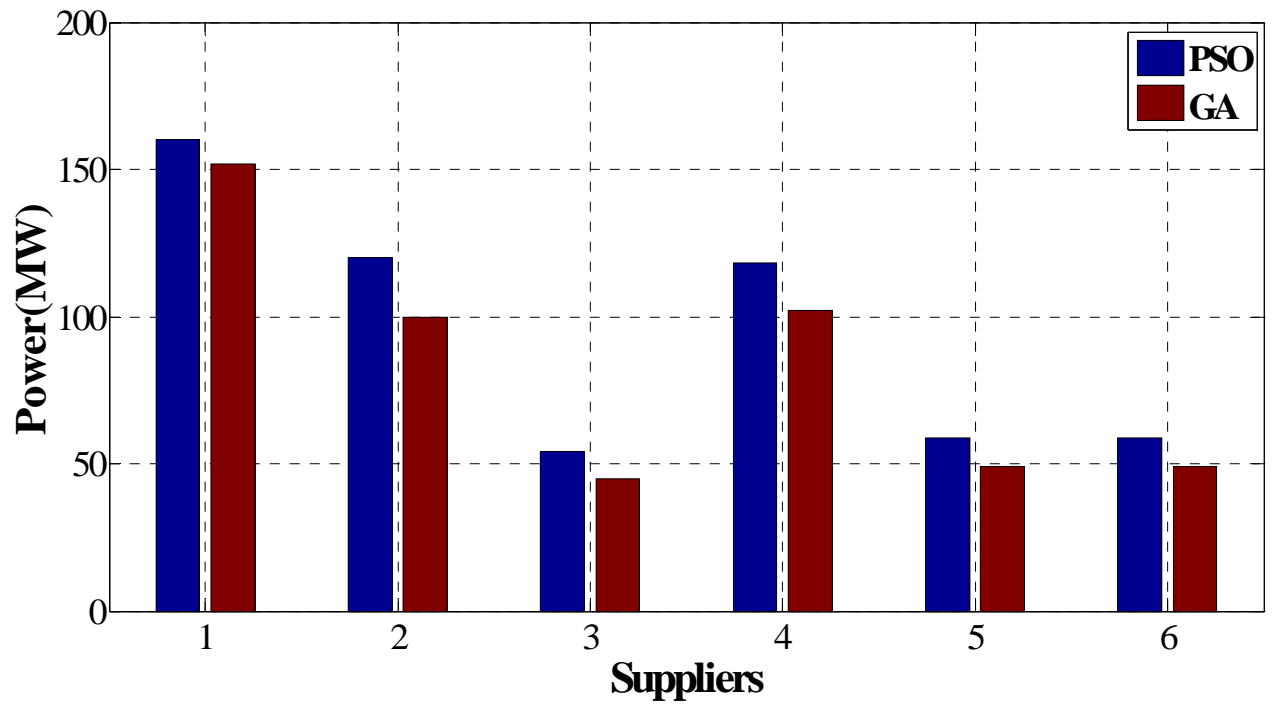

Figure 4 Expected dispatched powers of suppliers

4.2 Case B: With unsymmetrical information: In this case some participants to make better estimates than others could be included in the model. We now describe simulation results for an unsymmetrical situation in which the second supplier has less accurate estimates than the others. The second supplier's estimated expectation values of $a_{i}$ and $b_{i}$, i.e. and for $i=1,3,4,5,6$, are specified to be $1.4 e_{i}$ and $1.4 \times 2 f_{i}$, respectively, while the estimates of the other five suppliers and two consumers are the same as (13) and (14), respectively, and all other relevant parameters are the same as in Case A. by using this information, biding parameters, market clearing price, expected profit and expected dispatch of generators and demand of consumers are calculated using proposed method and compared with GA approach, are shown in Table 4 and Table 5. By comparing the results of Case A and case B, in Case B the second supplier moves up the market clearing price by making higher estimates about the bids of rival suppliers and hence offering a higher bid. These optimal strategies lead to an overall increase in benefits for all suppliers, but supplier 2, who has worse information than the others, suffers a benefit reduction. Figure 5 shows the values of expected profits of generators and demand of consumers obtained by using proposed method for Case A and Case B, and it is observed that the overall increase in benefit of all the suppliers except supplier 2, who has worse information than the others, suffers benefit reduction. 
Table 4. Bidding Strategies of Generators and Consumers

\begin{tabular}{|c|c|c|c|}
\hline & PSO & GA & $\begin{array}{c}\text { Monte Carlo } \\
\text { (David et al, 2001) }\end{array}$ \\
\hline Generator & $b_{i}$ & $b_{i}$ & $b_{i}$ \\
\hline 1 & 0.062 & 0.058 & 0.0297 \\
\hline 2 & 0.0964 & 0.182 & 0.1536 \\
\hline 3 & 0.243 & 0.221 & 0.292 \\
\hline 4 & 0.046 & 0.035 & 0.074 \\
\hline 5 & 0.124 & 0.116 & 0.170 \\
\hline 6 & 0.124 & 0.116 & 0.170 \\
\hline Large Consumer & $d_{j}$ & $d_{j}$ & $d_{j}$ \\
\hline 7 & 0.072 & 0.064 & 0.097 \\
\hline 8 & 0.051 & 0.049 & 0.077 \\
\hline
\end{tabular}

Table 5. Optimal Bid Prices (\$/MWh) and Profits (\$) of Generators and consumers

\begin{tabular}{|c|c|c|l|l|c|c|}
\hline & \multicolumn{2}{|c|}{ PSO } & \multicolumn{2}{c|}{ GA } & \multicolumn{2}{c|}{$\begin{array}{c}\text { Monte Carlo } \\
\text { (David et al, 2001) }\end{array}$} \\
\hline Generator & P (MW) & Profit & P (MW) & Profit & P (MW) & Profit \\
\hline 1 & 160.0 & 1705.24 & 160.00 & 1564.3 & 160.0 & 1411.3 \\
\hline 2 & 65.34 & 436.93 & 76.00 & 480.9 & 74.0 & 553.9 \\
\hline 3 & 56.60 & 480.02 & 48.60 & 386.1 & 46.6 & 336.9 \\
\hline 4 & 120.0 & 695.32 & 102.40 & 518.8 & 92.4 & 418.9 \\
\hline 5 & 59.27 & 310.06 & 47.70 & 232.8 & 44.7 & 190.8 \\
\hline 6 & 59.27 & 310.06 & 47.70 & 232.8 & 44.7 & 190.8 \\
\hline Large consumer & L (MW) & Profit & L (MW) & Profit & L (MW) & Profit \\
\hline 7 & 153.23 & 905.25 & 136.9 & 1092.4 & 136.9 & 1082.0 \\
\hline 8 & 120.97 & 388.07 & 108.6 & 562.7 & 108.6 & 556.1 \\
\hline MCP & \multicolumn{2}{|c|}{16.88} & \multicolumn{2}{c|}{16.74} & \multicolumn{3}{c|}{16.62} \\
\hline Total Profit & 5231.10 & \multicolumn{2}{c|}{5070.8} & 4739.8 \\
\hline
\end{tabular}

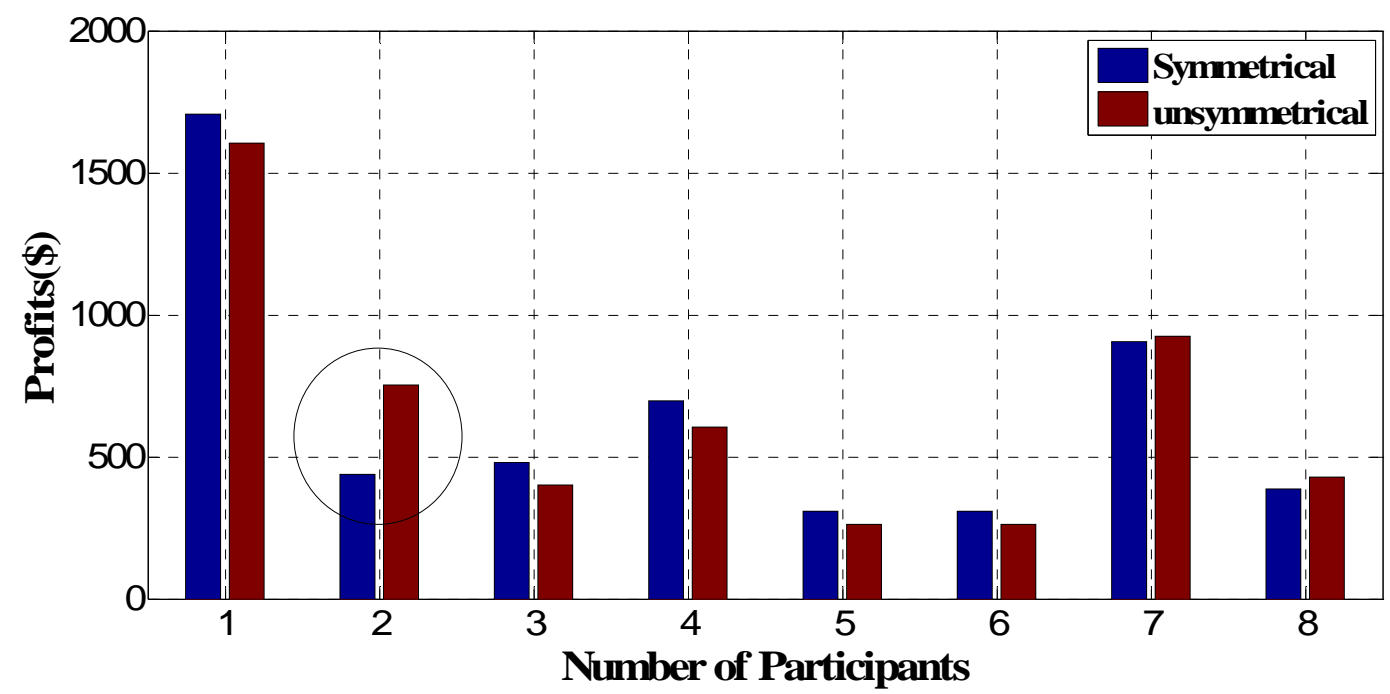

Figure 5 Expected profits of suppliers and consumers

The superiority of the PSO approach is demonstrated through comparison of simulation results with GA. Due to the randomness of the evolutionary algorithms, their performance cannot be judged by the result of a single run. Many trails with different initializations should be made to reach a valid conclusion about the performance of the algorithms. An algorithm is robust, if it can guarantee an acceptable performance level under different conditions. Since PSO and GA random in nature therefore the bidding data was executed 20 times for all the approaches. The best, worst, average value, total profit and PD for the given data of symmetrical information are tabulated in Table 6.The percentage deviation (PD) is computed as follows: 


$$
\mathrm{PD}=\frac{(\text { Best }- \text { Worst })}{\text { Best }} \times 100
$$

Table 6. Performance Comparison of Different Approaches

\begin{tabular}{|c|c|c|c|}
\hline & & PSO & GA \\
\hline & Best(\$) & 4741.4 & 4554.2 \\
\cline { 2 - 4 } Total Profit (\$) & Worst(\$) & 4652.8 & 4286.6 \\
\cline { 2 - 4 } & Ave.(\$) & 4697.1 & 4420.4 \\
\cline { 2 - 4 } & PD (\%) & 0.018 & 0.058 \\
\hline \multicolumn{2}{|c|}{ Average c.p.u. time (sec) } & 6.24 & 12.28 \\
\hline
\end{tabular}

From the results it is observed that the PD is minimum for the proposed PSO method compared to GA, for the given test system and optimal bidding strategies obtained by PSO producing higher profits compared to GA. In addition to that, PSO shows good consistency by keeping small variation between the best and worst solution. In other words, the simulation results show that, the PSO algorithm converges to global solution has a shorter c.p.u. time and small percentage deviation because it has the advantage of dealing with fewer operators compared to GA.

\section{Conclusion}

In this paper, application of PSO for strategic bidding is proposed for suppliers and large consumers in an open electricity market. In this approach, each participant tries to maximize its profit with the help of information announced by system operator. Symmetrical and Unsymmetrical information of rivals are discussed and it is observed that, those who are having imperfect information will suffer profit reduction. Advantage of dealing with only one operator and ability to control convergence makes the PSO method more efficient and superior than GA. In this work, these advantages of PSO are also confirmed with the simulation results.

\section{References}

Ahmet. D. Yucekya, Jorge Valenzuela, Gerry Dozier, Strategic bidding in electricity market using PSO, Elect. Power Syst. Res., 2009, Vol 79, pp. 335-345.

Azadeh. A, S.F. Ghaderi, B. Pourvalikhan Nokhanandan and M. Shaikhalishahi: 'A new GA approach for optimal bidding Strategy viewpoint of profit maximization of a generation company’ Expert system with applications, 2011 In press.

Bajpai P, S.K. Punna and S.N.Singh.: 'Swarm-based strategic bidding in competitive electricity markets'. IET Gener, Transm. Dist. 2008, Vol. 2, pp. 175-184.

Berry GA, Hobbs BF, “Analyzing Strategic Bidding Behavior in Transmission Networks”. IEEE tutorial on game theory Applications in electric power markets, IEEE PES, 1999.pp7-32.

David, A.K., and Wen, F.: 'Strategic bidding in competitive electricity markets: a literature survey'. IEEE PES Summer Meeting, 2000, Vol. 4, pp. 2168-2173.

David, A.K., and Wen, F.: 'Optimal bidding strategies for competitive generators and large consumers', Electr. Power Syst. Res., 2001, 59, pp. 37-43.

David, A.K., and Wen, F.: 'Optimally co-ordinate bidding strategies in energy and ancillary service markets', IEE Proc., Gener. Trans. Distrib., 2002, Vol. 3, pp. 331-338.

David, A.K., and Wen, F.S.: 'Optimal bidding strategies and modeling of imperfect information among competitive generators', IEEE Trans. Power Syst., 2001, Vol. 1, pp. 15-21.

Ebrahim Hasan, D. Francisco, and Galiana: 'Fast computation of pure strategy Nash equilibrium in electricity markets cleared by Merit order’ IEEE Trans. Power Syst., 2010, 25, pp. 722-728.

Ferrero, R.W., Ramesh, V.C., and Shahidehpour, S.M.: Transaction analysis in deregulated power system using game theory', IEEE Trans. Power Syst., 1997, Vol. 3, pp. 1340-1347.

Fleten, S.-E., and Pettersen, E.: 'Constructing bidding curves for a price-taking retailer in the Norwegian electricity market', IEEE Trans. Power Syst., 2005, Vol. 2, pp. 701-708.

Gross, G., and Finlay, D.J.: 'Optimal bidding strategies in competitive electricity markets'. Proc. 12th Power System Computation Conf., August 1996, pp. 815-823.

Hobbs, B.F., and Helman, U.: ‘Complementarity-based equilibrium modeling for electric power markets' in Bunn, D. (Ed.): 'Modeling Prices in Competitive Electricity Markets' (Wiley, New York, 2004).

Jain A.K., S.C.Srivastava, Strategic Bidding and risk Assessment Using Genetic Algorithm in Electricity Markets, International Journal of Emerging Electric Power Systems, 2009, Vol. 10, pp.1-10

Jianhui Wang, Zhi Zhou, Audun Botterud.: 'An evolutionary game approach to analyzing bidding strategies in electricity markets 
With elastic demand’ Energy, 2011, 36, pp. 3459-3467.

Kanakasabhapathy .P, K. Shanti Swarup, Bidding Strategy for of Pumped-Storage plant in pool-based electricity market, Energy conversion and Management. 51(3) (2010) 572-579.

Kennedy, J., and Eberhart, R.: 'Particle swarm optimization’. Proc. IEEE Int. Conf. Neural Networks, 1995, Vol. 4, pp. 19421948.

Pereira, M.V., Granville, S., Fampa, M., Dix, R., and Barroso, L.A.: 'Strategic bidding under uncertainty: a binary expansion Approach’, IEEE Trans. Power Syst., 2005, Vol. 1, pp. 180-188.

Torre, S.D., Conejo, A.J., and Contreras, J.: 'Finding multi-period Nash equilibrium in pool-based electricity markets', IEEE Trans. Power Syst., 2004, Vol.1, pp. 643-651.

Torre, S., Arroyo, J.M., Conejo, A.J., and Contreras, J.: 'Price maker self-scheduling in a pool-based electricity market: a mixedInteger LP Approaches’, IEEE Trans. Power Syst., 2002, Vol.4, pp. 1037-1042.

Ugedo, A., Lobato, E., Franco, A., Rouco, L., Ferna'ndez-Caro, J., and Chofr, J.: 'Strategic bidding in sequential electricity Markets', IEE Proc. Gen. Trans. Distrib., 2006, Vol. 4, pp. 431-442.

Yoshida, H., Kawata, K., Fukuyama, Y., Takayama, S., and Nakanishi, Y.: ‘A particle swarm optimization for reactive power and Voltage control considering voltage security assessment’, IEEE Trans. Power Syst., 2000, Vol. 4, pp. 1232-1239

Zhang, D., Wang, Y., and Luh, P.B.: 'Optimization based bidding strategies in deregulated market'. Proc. IEEE PES Power Industry Computer Applications Conf., 1999, pp. 63-68.

\section{Biographical notes}

J.Vijaya Kumar received B. Tech. from J. N. T. U Kakinada in 2002, M.Tech from Maulana Azad National Institute of Technology (MANIT) Bhopal in 2005 and currently pursuing Ph.D. degree in Electrical Engineering at National Institute of Technology (NIT), Warangal. His current research interests include power system deregulation and artificial intelligence technique applications in power systems.

Dr. D. M. Vinod Kumar received his B.E. degree in electrical engineering, his M.E. degree in Power Systems Engineering from University College of Engineering, Osmania University, Hyderabad, Andhra Pradesh, India, and his Ph.D. degree in electrical engineering from the Indian Institute of Technology (IIT), Kanpur, India. At present, he is Professor of Electrical Engineering at the National Institute of Technology, Warangal. His current research interests include power system deregulation, artificial intelligence technique applications in power systems, power system stability, Energy Management Systems (EMS) and Flexible AC Transmission Systems (FACTS).

Received April 2011

Accepted January 2012

Final acceptance in revised form February 2012 